\section{Oxidativer Burst}

H. Baum ${ }^{1}$ und W. -R. Külpmann ${ }^{2}$

${ }^{1}$ Institut für Laboratoriumsmedizin, Mikrobiologie und Blutdepot, Regionale Kliniken Holding RKH GmbH, Ludwigsburg, Deutschland

${ }^{2}$ Hannover, Deutschland

Synonym(e) Respiratorischer Burst

Englischer Begriff respiratory burst

Definition Bildung von Sauerstoffradikalen (s. > Reaktive Sauerstoffspecies) durch stufenweise Reduktion von molekularem Sauerstoff in den Phagolysosomen und perizellulär bei neutrophilen Granulozyten und anderen Phagozyten.

Beschreibung In den neutrophilen Granulozyten und anderen Phagozyten ( $\triangleright$ Monozyten, $\triangleright$ Makrophagen) ist die Bil- dung von für viele Mikroorganismen toxischen Sauerstoffradikalen ein Hauptmechanismus der Infektabwehr. Durch Phagozytose werden Bakterien in die neutrophilen Granulozyten aufgenommen. Diese Phagosomen verschmelzen mit den azurophilen Granula ( $\triangleright$ Granula, azurophile) zum Phagolysosom. Im Phagolysosom wird durch die NADPH-Oxidase und Cytochrom b ein freies Elektron auf molekularen Sauerstoff übertragen. Das entstehende $\mathrm{O}_{2}{ }^{-}$reagiert unter anderem mit $\mathrm{H}_{2} \mathrm{O}$ zu $\mathrm{H}_{2} \mathrm{O}_{2}$ und weiter, katalysiert durch die > Myeloperoxidase, mit $\mathrm{Cl}^{-}$zu hypochlorämischer Säure ( $\mathrm{HOCl}$ ). Zusätzlich entstehen OH-Radikale und Singlet-Sauerstoff ${ }^{1} \mathrm{O}_{2}$. All diese Produkte sind für viele Bakterien toxisch. Summativ werden diese Reaktionen als oxidativer Burst bezeichnet (s. a. > Oxidativer Burst-Test im Blut).

\section{Literatur}

Morel F, Doussiere J, Vignais PV (1991) The superoxide-generating oxidase of phagocytic cells. Eur J Biochem 201:523-546 